\title{
Use of Pyrimidine and Its Derivative in Pharmaceuticals: A Review
}

\section{Avinash U. Nerkar* Nashik-423 204, India. \\ ART T C LEDETAILS}

Department of Chemistry, Mahatma Gandhi Vidyamandir's SPH Arts, Science and Commerce College, Affiliated to Savitribai Phule Pune University, Nampur,

\section{Article history:}

Received 08 May 2021

Accepted 27 May 2021

Available online 25 June 2021

\section{Keywords:}

Pyrimidine

Antithyroid

Antiangiogenic

\begin{abstract}
A B S T R A C T
The pharmacological activities of the pyrimidine nucleus were impressive. Compounds with a pyrimidine nucleus have a broad variety of pharmaceutical applications, including antiviral, antibacterial, anti-inflammatory, sedatives and hypnotics, antidepressant, anticonvulsant, anti-thyroid, anti-Alzheimer and, according to the literature. As a result, the focus of this review is on research on various pharmaceuticals activities of pyrimidine analogs that has recently been published in the scientific literature.
\end{abstract}

\section{Introduction}

Pyrimidine derivatives are well-known in medicinal chemistry for their therapeutic applications. One potential explanation for their activity is the inclusion of a pyrimidine base in thymine, cytosine, and uracil, which are the basic binding blocks of nucleic acids, DNA, and RNA [1]. Pyrimidine analogs have previously been shown to be platelet aggregation inhibitors, antagonists, anti-conception, and anti-parkinsonism agents [2].

According to the literature, compounds with pyrimidine nuclei have a wide variety of biological activities like 5 -fluorouracil is an anticancer drug; idoxuridine and trifluoridine are antiviral drugs; zidovudine and stavudine are anti-HIV drugs; trimethoprim, sulphamethiazine, and sulphadiazine are antibacterial drugs. sulphadoxin is an antimalarial and antibacterial drug; minoxidil and prazosin are antihypertensive drugs. Barbiturates, such as phenobarbitone, are used as sedatives, hypnotics, and anticonvulsants. Propylthiouracil is used as an antithyroid. Because of its inclusion in a variety of natural products and structurally complex synthetic derivatives, the pyrimidine center has received a lot of attention. Intensive study has been focusing on the biological function of the pyrimidine nucleus as a result of the extraordinary pharmacological efficacy of pyrimidine derivatives [3-4]. Pyrimidine and the pyrimidine fused with naphtha structure is a field of pure and applied chemistry that is relatively new and quickly expanding. Structure, synthesis, spectral studies, bonding with a wide range of motifs and ligands, and their diverse reactivity in a number of fields Because of its unique electronic properties and wide range of uses, researchers are especially interested in its environmentally friendly synthesis [5-9]. Various correspondences have been based on the biological function of pyrimidine in recent years. Heterocyclic compounds have been studied for a wide range of biological properties [10-18].

\section{Anti-Viral Properties of Pyrimidine Derivatives}

Acyclic nucleoside phosphonates (ANPs) are important compounds with a wide range of biological activities, mainly antiviral activity. Krecmerovaet al. synthesized two new 2,4-diamino-6-[2(phosphonomethoxy)ethoxy]pyrimidine (PMEO-DAPy) (Fig. 1) and 1-[2(phosphonomethoxy) ethyl]-5-azacytosine (PME-5-azaC) (Fig. 2) prodrugs with a pro-moiety containing of carbonyloxymethyl esters (POM, 
cell lines such as breast cancer (MCF-7), lung cancer (A-549), colon cancer (Colo-205) and ovarian cancer (A2780). Against all cell lines studied, the majority of the screened compounds displayed moderate to excellent anticancer activity.

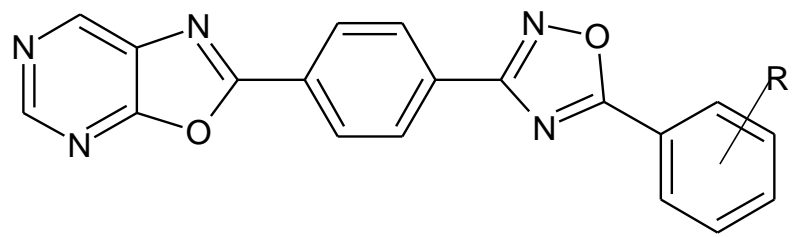

Fig. 3 Structure of 1,2,4-oxadiazole linked 4-(oxazolo[5,4-d]pyrimidine

\section{Anti-Fungal Properties of Pyrimidine Derivatives}

Fused pyrimidine derivatives, especially pyrido[2, 3-d] pyrimidine derivatives, have attracted a lot of attention among pyrimidine-containing compounds because they have interesting bioactivities including antifungal activity [22].

Acosta et al. prepared pyrazolo[4,3:5,6]pyrido[2,3-d]pyrimidine (Fig. 4) by a solvent-free microwave aided reaction of heterocyclic o-amino nitriles and cyano pyridines in the presence of tBuOK as catalyst. This protocol offers an innovative method for the synthesis of the compounds, with the benefits of simple set-up, mild reaction conditions, and high yields. All of the compounds were also screened for antifungal properties against Candida albicans and Cryptococcus neoformans, two clinically essential fungi. The most successful compounds were tested against a larger panel of clinical isolates in order to determine their actual efficacy against fungi from culture collections as well as patients with fungal infections [22].<smiles>[R]c1ccc(-c2c3c(N)nc(-c4cccnc4)nc3nc3c2c(C)cn3-c2ccccc2)cc1</smiles>

Fig. 4 Structure of pyrazolo[4,3:5,6]pyrido[2,3-d]pyrimidines

\section{Anti-Malarial Properties of Pyrimidine Derivatives}

Resulting in rapid emergence of multidrug resistant Plasmodium falciparum parasites, treating malaria has become a growing therapeutic problem. Plasmodium falciparum is responsible for having the most serious cases in humans, including death. Chloroquine and the pyrimethamine or sulfadoxine formulation used to be the first-line medicines for malaria control and prevention [23].

Pretorius et al. synthesized a sequence of quinoline-pyrimidine hybrids (Fig. 5) and assess their antimalarial activity and cytotoxicity in vitro. A two-step nucleophilic substitution process involving quinoline and pyrimidine moieties was used to create the hybrids.<smiles>Nc1cc(N2CCN(c3ccnc4cc(Cl)ccc34)CC2)nc(N)n1</smiles>

Fig. 5 Structure of quinoline-pyrimidine hybrid https://doi.org/10.30799/jacs.239.21070203
They were tested against Plasmodium falciparum D10 and Dd2 strains alongside chloroquine (CQ), pyrimethamine (PM), and set combinations of the two. The cytotoxicity was tested on the Chinese Hamster Ovarian cell line in mammals. Both strains were resistant to the compounds. The samples were screened in triplicate against Plasmodium falciparum strains that were chloroquine-susceptible (CQS) D10 and chloroquine-resistant (CQR) Dd2 continuous in vitro colonies of $P$. falciparum a sexual erythrocyte levels [23].

\section{Anti-Depressants and Anti-Convulsants Properties of Pyrimidine Derivatives}

Antidepressants and anticonvulsants are two of the most commonly used medications for CNS problems. The anticonvulsant and antidepressant actions of a sequence of 5-alkoxytetrazolo [1, 5-c] thieno $[2,3-e]$ pyrimidine derivatives were investigated. At a dosage of 100 $\mathrm{mg} / \mathrm{kg}$, the most active compound was 5-(2,4dichlorobenzyloxy)tetrazolo [1,5-c] thieno[2,3-e]pyrimidine, which reduced immobility period by 51.62 percent [24].

A series of 5-alkoxytetrazolo [1,5-c]thieno[2,3-e]pyrimidine (Fig. 6) derivatives were synthesized by Wang et al. Their anticonvulsant activities and antidepressant activities were used to assess the maximal electroshock (MES) and forced swimming tests (FST).<smiles>[R]c1nc2ccsc2c2nnnn12</smiles>

Fig. 6 Structure of 5-alkoxytetrazolo [1,5-c]thieno[2,3-e]pyrimidine

\section{Anti-Thyroid Properties of Pyrimidine Derivatives}

Lacotte et al. synthesized and evalutate the first and rate-limiting step in the biosynthesis of the iodinated hormones T3 and T4 is iodide translocation into thyroid cells. The sodium iodide symporter is involved in this operation (NIS), A glycoprotein with 13 putative trans membrane domains that is primarily found in the thyroid gland but also in other tissues during lactation, such as salivary glands, gastric mucosa, and mammary glands. The efficacy of dihydropyrimidin-2-ones (DHPMs) (Fig. 7) to inhibit iodide entrapment in rat thyroid cells was investigated. The multicomponent Biginelli reaction was used to complete the synthesis. In a cell-based experiment, twelve compounds were evaluated for their ability to inhibit the sodium iodide symporter (NIS). With a half maximum inhibitory concentration value (IC50) of $65 \mathrm{pM}$, one newly synthesized derivative showed unusually strong behavior. This research adds to the advancement of anti-thyroid drug [25].<smiles>CC1=C(C(=O)OCc2ccc3c(c2)OCO3)C(c2ccco2)N(C)C(=O)N1C</smiles>

Fig. 7 Structure of dihydropyrimidin-2-ones

\section{Anti-Inflammatory Properties of Pyrimidine Derivatives}

As of emerging infectious diseases and the number of multidrugresistant microbial pathogens, treating bacterial infections remains a difficult therapeutic issue. Despite the abundance of antibiotics and chemotherapeutics, the proliferation of old and modern antibioticresistant bacterial strains in recent decades has necessitated the development of new antibacterial groups [26]. 
Keche et al. synthesized a series of new 4-(3(trifluoromethyl)phenylamino-6-(4-(3-arylureiodo/arylthioureido/ arylsulfonamido)-pyrimidine derivatives (Figs. 8 and 9) of biological interest were prepared by the sequential Suzuki cross coupling, acid amination, reduction followed by reaction of resulting amine with different arylisocyantes or arylisothiocyantes or arylsulfonyl chlorides. The antimicrobial activity and pro-inflammatory cytokines (TNF-a and IL6) of the synthesized compounds were tested (antibacterial and antifungal) [26].

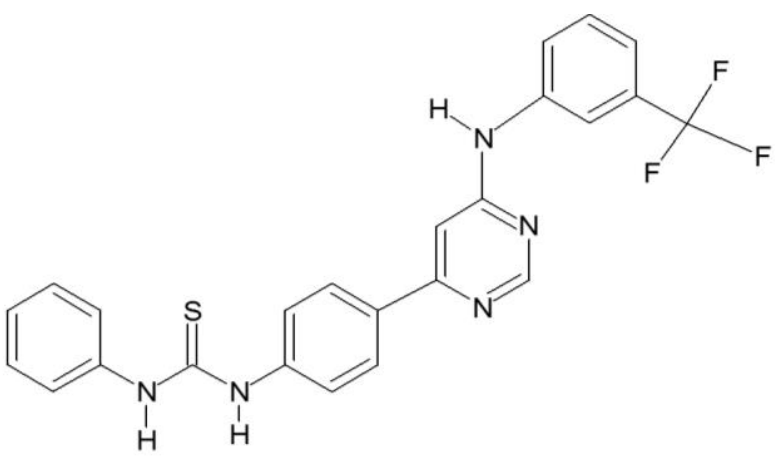

Fig. 8 Structure of 4-(3-(trifluoromethyl)phenylamino-6-(4-(3-arylthioureido)pyrimidine derivative<smiles>CC1=CCCC(F)=C1S(=O)(=O)Nc1ccc(-c2cc(Nc3cccc(C(F)(F)F)c3)ncn2)cc1</smiles>

Fig. 9 Structure of 4-(3-(trifluoromethyl)phenylamino-6-(4-(3-arylsulfonamido)pyrimidine derivative

\section{Anti-Alzheimer Properties of Pyrimidine Derivatives}

Alzheimer disease (AD) is a neurodegenerative disease that causes memory loss and cognitive impairment. Representative histopathological findings in $\mathrm{AD}$ brain include extracellular amyloid- $\beta$ (A $\beta$ ) plaques, intracellular neurofibrillary tangles composed of hyperphosphorylated tau, and a continuous loss of neurons. Many people with late-onset Alzheimer's disease have a respiratory complex IV function deficiency. Upstream from respiratory complex IV, the de novo pyrimidine biosynthesis pathway interacts with the mitochondrial respiratory chain [27]. Pesini et al. predicted that these patients' pyrimidine nucleotide levels will be lower. Different cell processes that rely on these chemicals, such as neuronal membrane generation and maintenance, as well as the formation of synapses, will be jeopardized. They proved that inhibiting oxidative phosphorylation activity decreases neuronal differentiation using a cell model. Uridine therapy restores neuronal distinction by linking these pathways to pyrimidine nucleotides. In the brains of Alzheimer's patients, we found altered mRNA levels for genes involved in both de novo pyrimidine biosynthesis and pyrimidine salvage pathways [27].

\section{Anti-Angiogenic of Pyrimidine Derivatives}

Angiogenesis is a natural mechanism for organ production that involves the creation of new blood vessels by forming new capillaries from established vasculature. Angiogenesis can be involved in the formation and progression of diseases including rheumatoid arthritis, inflammation, ocular neovascularization, psoriasis, tumor proliferation, and metastasis where the regulating mechanisms of angiogenesis are disrupted. This mechanism involves more than twenty distinct causes, one of which is vascular endothelial growth factors (VEGFs). VEGF-A (also known as VEGF), VEGF-B, VEGF-C, VEGF-D, VEGF-E, VEGF-F, and Placental Growth Factor (PlGF) are all members of the VEGF family [28]. Perspicace et al. https://doi.org/10.30799/jacs.239.21070203 formulated, synthesized, and tested thieno pyrimidines for their ability to inhibit VEGFR-2. Compound (Fig. 10) was identified as the lead compound of all the synthesized compounds because it inhibits VEGFR-2 and HUVEC at very low concentrations. In vitro experiments showed that tartaric acid salt of compound (10) $(\mathrm{EC} 50=31 \mathrm{nM})$ inhibited endothelial cell tube development caused by VEGF, whereas standard drug Sunitinib $(E C 50=645 \mathrm{nM})$ did not. As a result, it may be used as a reference point for the synthesis of anti-angiogenic agents.<smiles>O=c1[nH]c(CN2CCCCC2)nc2cc(-c3cn(Cc4ccccc4)c4ccccc34)sc12</smiles>

Fig. 10 Structure of Thieno pyrimidines

\section{Anti-Hepatitis Properties of Pyrimidine Derivatives}

In humans, hepatitis B virus (HBV) and hepatitis $\mathrm{C}$ virus (HCV) are the most common causes of chronic liver disease. HBV and HCV co-infection is normal, and it's linked to an elevated risk of liver cancer, cirrhosis, and hepatocellular carcinoma, both of which can lead to death [29]. The synthesis and anti-HCV action of a new class of pyrimidine nucleosides with a 4-carboxymethyl and 4-carboxamide functional group were stated by Shakya et al. Any of the compounds were found to be effective anti-HCV agents with no toxicity. The findings showed that these compounds had anti-HCV functions that were equivalent to ribavirin $(\mathrm{EC50}=81.9 \mathrm{M})$.The most active analog of the fully synthesized compounds, (Fig. 11) was discovered to interact synergistically with ribavirin to inhibit HCV RNA replication [29].

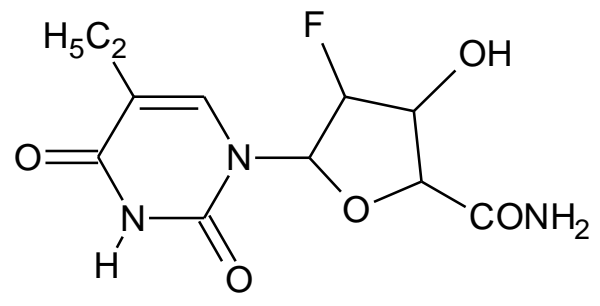

Fig. 11 Structure of $4^{\prime}$-carboxamide pyrimidine nucleoside

\section{Conclusion}

This study brings to light the pyrimidine derivatives and their diverse potential in drug development and medicine. It is evident that pyrimidine derivatives have been investigated for a number of ailments as highlighted in this review. Although pyrimidine and its derivatives are reported to have diverse pharmacological activities and their role, as antiinflammatory, anticancer, antiviral, anticonvulsant agents, etc. It is very useful compound in pharmaceutical industry. There is still scope for more research work to be done in this field to find a novel pharmaceutical activity. Furthermore, due to the resistance of currently available drugs, there is a strong need to pursue further research on pyrimidine derivatives.

\section{Acknowledgment}

The author acknowledge the Department of Chemistry, Mahatma Gandhi Vidyamandir's SPH Arts, Science and Commerce College, Nampur District, Nashik, Maharashtra, India.

\section{References}

[1] M.E. Sahu, N.A. Siddiqui, A review on biological importance of pyrimidines in the new era, Int. J. Pharm. Pharm. Sci. 8(5) (2016) 8-21.

[2] N.O. Al-Harbi, S.A. Bahashwan, A.A. Fayed, M.S. Aboonq, E.E.E. Amr, Antiparkinsonism, hypoglycemic and anti-microbial activities of new poly fused ring heterocyclic candidates, Int. J. Biol. Macromol. 57(1) (2013) 65-73.

[3] T.P. Selvam, C.R. James, P.V. Dniandev, S.K. Valzita, A mini review of pyrimidine and fused pyrimidine marketed drugs, Res. Pharm. 2(4) (2012) 1-9. 
[4] R.S. Mohana, R. Sompalle, Synthetic chemistry of pyrimidines and fused pyrimidines: a review, Synth. Commun. 46(8) (2016) 645-672.

[5] V.A. Adole, R.H. Waghchaure, S.S. Pathade, M.R. Patil, T.B. Pawar, B.S. Jagdale, Solvent-free grindstone synthesis of four new (E)-7-(arylidene)-indanones and their structural, spectroscopic and quantum chemical study: a comprehensive theoretical and experimental exploration, Mol. Simul. 46(14) (2020) 10451054.

[6] V.A. Adole, B.S. Jagdale, T.B. Pawar, A.A. Sagane, Ultrasound promoted stereoselective synthesis of 2,3-dihydrobenzofuran appended chalcones at ambient temperature, S. Afr. J. Chem. 73 (2020) 35-43.

[7] R.A. Shinde, V.A. Adole, B.S. Jagdale, T.B. Pawar, B.S. Desale, R.S. Shinde, Efficient synthesis, spectroscopic and quantum chemical study of 2, 3dihydrobenzofuran labelled two novel arylidene indanones: A comparative theoretical exploration, Mat. Sci. Res. India 17(2) (2020) 146-161.

[8] V.A. Adole, Synthetic approaches for the synthesis of dihydropyrimidinones/ thiones (biginelli adducts): a concise review, World J. Pharm. Res. 9(6) (2020) 1067-1091.

[9] V.A. Adole, P.B. Koli, R.A. Shinde, R.S. Shinde, Computational insights on molecular structure, electronic properties, and chemical reactivity of (E)-3-(4chlorophenyl)-1-(2-hydroxyphenyl)prop-2-en-1-one, Mat. Sci. Res. India 17 (2020) 41-53.

[10] V.A. Adole, B.S. Jagdale, T.B. Pawar, B.S. Desale, Molecular structure, frontier molecular orbitals, MESP and UV-visible spectroscopy studies of Ethyl 4-(3, 4dimethoxyphenyl)-6-methyl-2-oxo-1,2,3,4-tetrahydropyrimidine-5carboxylate: A theoretical and experimental appraisal, Mat. Sci. Res. India 17 (specialissue2020) (2020) 13-36.

[11] R.A. Shinde, V.A. Adole, Anti-microbial evaluation, Experimental and theoretical insights into molecular structure, electronic properties, and chemical reactivity of (E)-2-((1H-indol-3-yl) methylene)-2, 3-dihydro-1Hinden-1-one, J. Appl. Organomet. Chem. 1(2) (2021) 48-58.

[12] H.H. Gong, D. Addla, J.S. Lv, C.H. Zhou, Heterocyclic naphthalimides as new skeleton structure of compounds with increasingly expanding relational medicinal applications, Curr. Top. Med. Chem. 16(28) (2016) 3303-3364.

[13] V.A. Adole, Synthesis, antibacterial, antifungal and DFT studies on structural, electronic and chemical reactivity of (E)-7-((1H-Indol-3-yl) methylene)-1, 2, 6, 7-tetrahydro-8H-indeno [5, 4-b] furan-8-one, Adv. J. Chem. A 4(3) (2021) 175187.

[14] R.A. Shinde, V.A. Adole, B.S. Jagdale, T.B. Pawar, Superfast synthesis, antibacterial and antifungal studies of halo-aryl and heterocyclic tagged 2, 3dihydro-1 H-inden-1-one candidates, Monatsh. Chem. Chemical Monthly 152 (2021) 649-658

[15] R.A. Shinde, V.A. Adole, B.S. Jagdale, B.S. Desale, Synthesis, antibacterial and computational studies of halo chalcone hybrids from 1-(2, 3-dihydrobenzo [b][1, 4] dioxin-6-yl) ethan-1-one, J. Ind. Chem. Soc. 98(4) (2021)100051.

[16] V.A. Adole, R.H. Waghchaure, B.S. Jagdale, T.B. Pawar, S.S. Pathade, Molecular structure, frontier molecular orbital and spectroscopic examination on dihydropyrimidinones: a comparative computational approach, J. Adv. Sci. Res. 11(2) (2020) 64-70.

[17] R.S. Shinde, Ultrasound assisted synthesis, molecular structure, UV-visible assignments, MEP and Mulliken charges study of (E)-3-(4-chlorophenyl)-1-(4methoxyphenyl) prop-2-en-1-one: experimental and DFT correlational, Mat. Sci. Res. India 18(1) (2021) 86-96.

[18] R.S. Shinde, Synthesis, characterization and theoretical insights into molecular properties of 2-(4-fluorophenyl)-4H-chromen-4-one, J. Adv. Chem. Sci. 7(2) (2021) 721-724.

[19] M. Krecmerova, M. Dracinsky, R. Snoeck, J. Balzarini, K. Pomeisl, G. Andrei, New prodrugs of two pyrimidine acyclic nucleoside phosphonates: Synthesis and antiviral activity, Bioorg. Med. Chem. 25(17) (2017) 4637-4648.

[20] B. Kumar, P. Sharma, V.P. Gupta, M. Khullar, S. Singh, N. Dogra, V. Kumar, Synthesis and biological evaluation of pyrimidine bridged combretastatin derivatives as potential anticancer agents and mechanistic studies, Bioorg. Chem. 78 (2018) 130-140.

[21] N. Perupogu, D.R. Kumar, D. Ramachandran, Anticancer activity of newly synthesized 1,2,4-oxadiazole linked 4-oxazolo[5,4-d]pyrimidine derivatives, Chem. Data Collect. 27 (2020) 100363.

[22] P. Acosta, B. Insuasty, A. Ortiz, R. Abonia, M. Sortino, A.S. Zacchino, J. Quiroga Solvent-free microwave-assisted synthesis of novel pyrazolo $\left[4^{\prime}, 3^{\prime}: 5,6\right]$ pyrido[2,3-d]pyrimidines with potential antifungal activity, Arab. J. Chem. 9(3) (2016) 481-492.

[23] S.I. Pretorius, W.J. Breytenbach, C. De Kock, P.J. Smith, D. David, Synthesis, characterization and antimalarial activity of quinoline-pyrimidine hybrids, Bioorg. Med. Chem. 21(1) (2013) 269-277.

[24] S. Wang, X.Q. Deng, Y. Zheng, Y.P. Yuan, Z.S. Quan, L. Guan, Synthesis and evaluation of anticonvulsant and antidepressant activities of 5alkoxytetrazolo[1,5 c] thieno[2,3-e]pyrimidine derivatives, Eur. J. Med. Chem. 56 (2012) 139-144.

[25] P. Lacotte, D. Buisson, Y. Ambroise, Synthesis, evaluation and absolute configuration assignment of novel dihydropyrimidin-2-ones as picomolar sodium iodide symporter inhibitors, Eur. J. Med. Chem. 62 (2013) 722-727.

[26] A.P. Keche, G.D. Hatnapure, R.H. Tale, A. H. Rodge, S.S. Birajdar, V.M. Kamble, A novel pyrimidine derivatives with aryl urea thiourea and sulfonamide moieties: Synthesis, anti-inflammatory and antimicrobial evaluation, Bioorg. Med. Chem. Lett. 22(10) (2012) 3445-3448.

[27] A. Pesini, E. Iglesias, M.P.B. Bafaluy, N.G. Perez, P. Meade, et al., Brain pyrimidine nucleotide synthesis and Alzheimer disease, Aging (Albany NY) 11(19) (2019) 8433-8462.

[28] E. Perspicace, V. Jouan-Hureaux, R. Ragno, F. Ballante, S. Sartini, C.L. Motta Design, synthesis and biological evaluation of new classes of thieno [3,2-d] pyrimidinone and thieno[1,2,3]triazine as an inhibitor of vascular endothelial growth factor receptor-2 (VEGFR-2), Eur. J. Med. Chem. 63 (2013) 765-781.

[29] N. Shakya, S. Vedi, C. Liang, F. Yang, B. Agrawal, R. Kumar R, 4'-Substituted pyrimidine nucleosides lacking 5'-hydroxyl function as potential anti-HCV agents, Bioorg. Med. Chem. Lett. 24 (2014) 1407-1409. 